\title{
Unexpected amyloidosis, mimicking a splenic cyst
}

\author{
Chao-Feng Chang ${ }^{1}$, Yi-Shu Liao ${ }^{2}$, Yu-Guang Chen ${ }^{3}$, and Ren-Hua Ye ${ }^{3}$
}

Departments of ${ }^{1}$ Internal Medicine and ${ }^{2}$ Pathology, ${ }^{3}$ Division of Haematology and Oncology, Department of Internal Medicine, Tri-Service General Hospital, National Defence Medical Centre, Taipei, Taiwan

Received: April 17, 2016

Revised: July 2, 2016

Accepted: October 19, 2016

\section{Correspondence to}

Ren-Hua Ye, M.D.

Tel: +886-921035129

Fax: +886-287927209

E-mail: minceing@yahoo.com.tw
An 87-year-old male presented to our hospital with a low-grade fever and left upper quadrant abdominal pain for 1 week. Laboratory investigations revealed the white blood cell count to be 13,280/ $\mathrm{UL}$ (normal value, 4.50 to $11.00 \times 10^{3} / \mu \mathrm{L}$ ); hemoglobin $12.8 \mathrm{~g} / \mathrm{dL}$ (normal value, 13.5 to 18.0); and platelet count, 115,000/UL (normal value, 150 to $400 \times 10^{3} / \mu \mathrm{L}$ ). Furthermore, aspartate transaminase levels were $18 \mathrm{U} / \mathrm{L}$ (normal value, < 40); alanine transaminase $20 \mathrm{U} / \mathrm{L}$ (normal value, $<41$ ); creatine, $1.2 \mathrm{mg} / \mathrm{dL}$ (normal value: $0.7-1.2$ $\mathrm{mg} / \mathrm{dL}$ ); and C-reactive protein $2.8 \mathrm{mg} /$ $\mathrm{dL}$ (normal value, < o.8). An abdominal sonography revealed a heterogeneous, hypoechoic mass, measuring about 6.6 $\times 6.3 \mathrm{~cm}$ in size, in the left subphrenic space. Based on these clinical manifestations and laboratory results, an initial diagnosis of splenic cyst due to unknown etiology was made. Broad-spectrum antibiotics were administrated as initial therapy due to a suspected splenic abscess. However, a follow-up computed tomography (CT) of the abdomen showed a heterogeneous attenuation (about $6.2 \times 6.3 \mathrm{~cm}$ ) in size with mural nodules in the spleen (Fig. 1). Meanwhile, the blood culture and cardiac echography findings were negative for distant hematologic spread. Additional investigations performed for a suspected splenic infarction, including D-Dimer, immunoglobulin G (IgG) anti-cardiolipin antibody, IgM anti-cardiolipin antibody, lupus anticoagulant, anti-nuclear antibodies
$\mathrm{C}_{3}$ and $\mathrm{C}_{4}$, all revealed unremarkable findings. However, the patient underwent a sonography-guided drainage of the spleen due to the failure of the first-line therapy. The abscess culture revealed sterile findings. Microscopic examination revealed many plasma cells surrounding the amyloid-like deposition (Fig. 2A) as well as polyclonality of $\kappa$ and $\lambda$ light chains. The immunostaining was thioflavin $\mathrm{S}$ positive under high-background fluorescence (Fig. 2B). Serum immunoelectrophoresis revealed monoclonal proliferation of immunoglobulins (IgG, 1,670 mg/ dL). No obvious M-protein was detected on serum protein electrophoresis. The $\lambda$ serum free light chain concentration was $31.40 \mathrm{mg} / \mathrm{L}$ (normal value, 5.71 to 26.30); $\kappa$ serum free light chain concentration was $23.30 \mathrm{mg} / \mathrm{L}$ (normal values, 3.30 to 19.40 ), and the $\kappa / \lambda$ ratio was 0.74 . The 24 -hour urine $\kappa$ and $\lambda$ concentrations were 313.6 and $1,328 \mathrm{mg} /$ day, respectively; the urine $\kappa / \lambda$ ratio was 0.236 (normal value, 0.26 to 1.65 ). Cardiac echography demonstrated a preserved ejection fraction of the left and right ventricles. Furthermore, no wall thickness or abnormal myocardial texture was observed. There were no particular symptoms related to organ involvement, such as hepatomegaly, peripheral neuropathy, and nephrotic range proteinuria. Thus, a final diagnosis of primary amyloid light-chain (AL) amyloidosis of the spleen was established. 


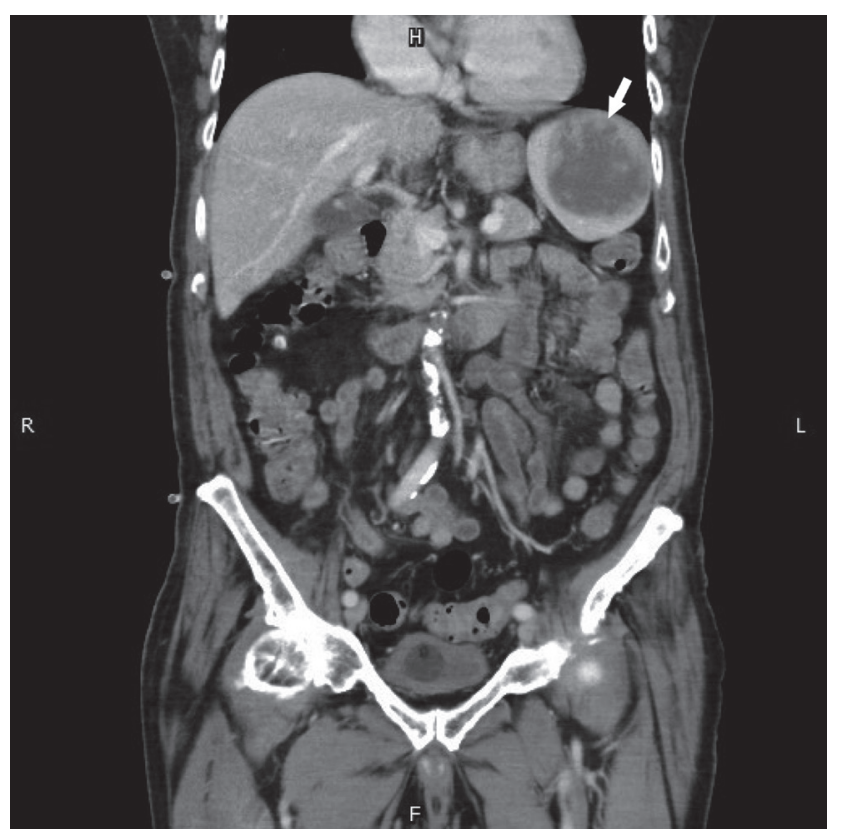

Figure 1. Heterogeneous attenuation (about $6.2 \times 6.3 \mathrm{~cm}$ in size, white arrow) with mural nodules in the spleen.

Among the differential diagnoses of a splenic cyst, primary causes account for $10 \%$ to $25 \%$ of cases, including congenital cysts and hematologic disorders, and secondary causes account for $80 \%$ cases, including trauma, abscess, and infarction with a liquefactive change. Asymptomatic splenic cyst is often unexpectedly detected on a radiologic study. A splenic cyst is usually spherical and well-demarcated with a low attenuation near water on CT, and sometimes, calcification and a septum are found in the cyst wall. However, in more than $70 \%$ of the patients splenic cyst is associated with symptoms

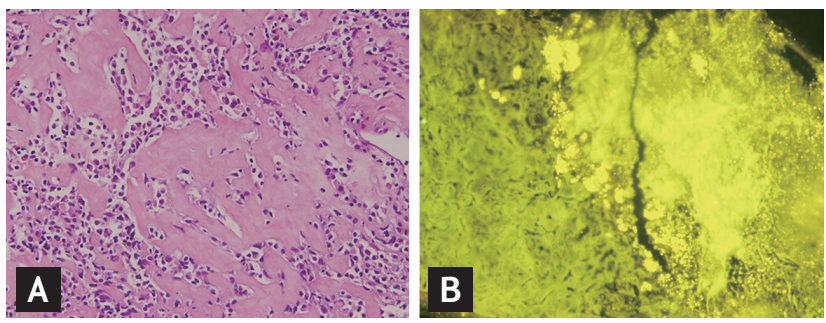

Figure 2. (A) Distinctive plasma cells surrounding the amyloid-like deposition $(\mathrm{H} \& \mathrm{E}, \times 400)$. (B) Positive thioflavin $\mathrm{S}$ staining (×400) under high-background fluorescence.

such as left upper quadrant abdominal pain, abdominal distension, or splenomegaly. The splenic cyst caused by an isolated splenic amyloidosis in the present case was a rare occurrence. The occurrence of a localized amyloidosis is up to $20 \%$, and splenic involvement is only $5 \%$ to $10 \%$. Most cases of splenic amyloidosis are caused by a systemic AL amyloid. Splenic amyloidosis is usually asymptomatic, but it may be accompanied by hepatic amyloidosis with manifestations of hepatomegaly and abnormal liver function. Clinicians should be aware that amyloidosis of the spleen can lead to hyposplenism with subsequent infectious complications; moreover, there is an increased risk for developing atraumatic spontaneous splenic rupture.

\section{Conflict of interest}

No potential conflict of interest relevant to this article was reported. 